\title{
NOTES
}

\section{Diffusion during the Immersion Precipitation Process}

\section{INTRODUCTION}

Polymer membranes can be obtained by the so-called immersion precipitation process in which a cast solution of a polymer in a solvent is immersed in a nonsolvent bath. The structure of the membranes produced in this manner is determined by two distinct factors: (i) the phase separation phenomena (equilibria and kinetics) in the ternary system, and (ii) the rate of diffusive solventnonsolvent exchange during the immersion.

Mechanisms of formation of synthetic membranes which incorporate these two factors have been proposed by several authors. ${ }^{1-8}$ In this note we comment on the mathematical description of the diffusion problem as suggested by Cohen, Tanny, and Prager ${ }^{6}$ in their paper "Diffusion-Controlled Formation of Porous Structures in Ternary Polymer Systems." This description includes a steady-state assumption which, in our point of view, is erroneous. Nevertheless, when this assumption is not applied, the paper of Cohen, Tanny, and Prager offers a valuable approach to the diffusion problem.

\section{THE MODEL}

Cohen, Tanny, and Prager ${ }^{6}$ propose a theory for the appearance of two-phase structures during the formation of polymer membranes from a casting solution immersed in a nonsolvent bath. This theory contains a series of assumptions, some concerning the phase separation phenomena and the others the diffusion phenomenon. Since this note deals with the diffusion problem, we summarize here the diffusion model.

(i) A schematic representation of the immersion process is given in Figure 1. At time $t=0$ the casting solution possesses its original volume fractions $\phi_{1}^{c}, \phi_{2}^{c}$, and $\phi_{3}^{c}$ (1 denotes a nonsolvent, 2 a solvent, and 3 a polymer). At time $t=t$ a diffusion layer has propagated to $z=z_{d}(t)$. The coordinate $z$ is a position coordinate in a laboratory frame. The volume fractions at the film surface are $\phi_{1}^{s}$, $\phi_{2}^{s}$, and $\phi_{3}^{s}$, which are considered to be at equilibrium with the coagulation bath. At time $t=t$ the film/bath interface has moved away from $z=0$ as a result of the net volume outflow.

(ii) A position coordinate is introduced, measured in terms of the volume $m$ of polymer per unit area of membrane between the interface and the point of observation':

$$
m(z)=\int_{0}^{z} \phi_{3}(z) \cdot d z
$$

or

$$
d m=\phi_{3} \cdot d z
$$

As a consequence, the film/bath interface is always at $m=0$. The position of the film/support interface on the $m$ axis, $M$, is also independent of time:

$$
M=\int_{0}^{Z} \phi_{3}(z) \cdot d z \equiv \phi_{3}^{c} \cdot Z
$$

(iii) The diffusion fluxes $J_{1}$ and $J_{2}$ through surfaces of fixed $m$ are assumed to be linearly related to the driving forces:

$$
J_{1}=-\frac{D_{1}\left(\phi_{1}, \phi_{2}\right)}{R T} \cdot \phi_{1} \cdot \frac{\partial \mu_{1}}{\partial m}, J_{2}=-\frac{D_{2}\left(\phi_{1}, \phi_{2}\right)}{R T} \cdot \phi_{2} \cdot \frac{\partial \mu_{2}}{\partial m}
$$

$D_{1}$ and $D_{2}$ are the diffusion coefficients for nonsolvent and solvent in the polymer-fixed reference frame and $\mu_{1}$ and $\mu_{2}$ are the chemical potentials. Cross terms have been neglected, but nevertheless eqs. (3) are interrelated through the expressions for $D_{1}, D_{2}, \mu_{1}$, and $\mu_{2}$. 


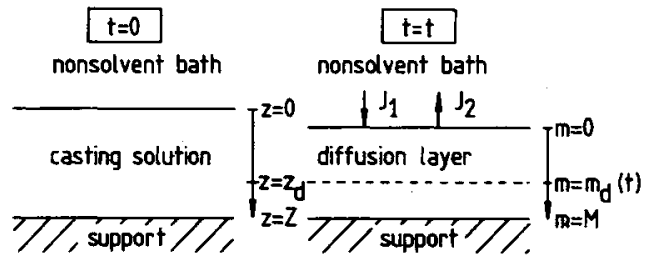

Fig. 1. Schematic representation of the immersion process at two different times. $J_{1}$ and $J_{2}$ are the nonsolvent and solvent volume flux, respectively. The coordinate $z$ is fixed; the coordinate $m$ is measured in terms of the polymer volume per unit area membrane between $z=0$ and the point of observation.

(iv) Fick's law, applied in the " $m$ " reference frame, gives (see Appendix A)

$$
\frac{\partial\left(\phi_{1} / \phi_{3}\right)}{\partial t}=-\frac{\partial J_{1}}{\partial m}, \frac{\partial\left(\phi_{2} / \phi_{3}\right)}{\partial t}=-\frac{\partial J_{2}}{\partial m}
$$

where $\phi_{3}=1-\phi_{1}-\phi_{2}$.

The initial and boundary conditions are:

$$
\begin{gathered}
t=0: \quad \phi_{1}(m, 0)=\phi_{1}^{c}, \phi_{2}(m, 0)=\phi_{2}^{c} \\
m=0: \quad \phi_{1}(0, t)=\phi_{1}^{s}, \phi_{2}(0, t)=\phi_{2}^{s} \\
m=M ; \quad J_{1}(M, t)=J_{2}(M, t)=0
\end{gathered}
$$

(v) Cohen, Tanny, and Prager restrict themselves to the case in which the diffusion layer has not yet reached the support. Thus, eq. (7) is replaced by

$$
m=m_{d}(t): \quad \phi_{1}\left(m_{d}, t\right)=\phi_{1}^{c}, \phi_{2}\left(m_{d}, t\right)=\phi_{2}^{c}
$$

(vi) Furthermore, they assume that the concentration distribution in the diffusion layer at every time is the steady-state distribution for a sheet of thickness $m_{d}(t)$ satisfying the boundary conditions (6) and (8). This implies that the fluxes $J_{1}$ and $J_{2}$ are independent of $m$ :

$$
\frac{\partial J_{1}}{\partial m}=\frac{\partial J_{2}}{\partial m}=0
$$

It should be noted that, owing to the steady-state assumption, eq. (4) and the initial condition (5) no longer have a bearing on the solution of the diffusion problem.

(vii) The diffusion problem is now reduced to eqs. (3). The ratio of $J_{1}$ and $J_{2}$ can be written as

$$
\frac{J_{1}}{J_{2}}=\sigma=\frac{\phi_{1}}{\phi_{2}} \cdot \frac{d \mu_{1}}{d \mu_{2}}
$$

The assumption made here is that the ratio $D_{1}\left(\phi_{1}, \phi_{2}\right) / D_{2}\left(\phi_{1}, \phi_{2}\right)$ is constant and unity. The ratio $\sigma$ normally has a negative value.

(vii) If the differentials of the chemical potentials are expressed as functions of the volume fractions, one finds

$$
\frac{d \phi_{2}}{d \phi_{1}}=\frac{\sigma \phi_{2}\left(\partial \mu_{2} / \partial \phi_{1}\right)-\phi_{1}\left(\partial \mu_{1} / \partial \phi_{1}\right)}{\phi_{2}\left(\partial \mu_{1} / \partial \phi_{2}\right)-\sigma \phi_{2}\left(\partial \mu_{2} / \partial \phi_{2}\right)}
$$

This first-order differential equation yields the relation between $\phi_{1}$ and $\phi_{2}$ in the diffusion layer as a function of the ratio $\sigma$ and one of the boundary conditions.

With the aid of the Flory-Huggins expressions ${ }^{10}$ for the chemical potentials together with eq. (11), Cohen, Tanny, and Prager calculated the composition paths within the ternary phase diagram and discussed them in relation to the formation of membranes.

\section{Critique of the Steady-State Assumption}

Equation (11) has been derived using the steady-state condition (9). In our view this condition can not be applied to this diffusion problem. The use of it neglects the initial condition (5) and therefore does not satisfy the mass conservation law. 
To make this point clear, the following example is considered. Equations (3) are rewritten as

$$
\begin{aligned}
& J_{1}=-\frac{D_{1}}{R T} \cdot \phi_{1} \cdot\left(\frac{\partial \mu_{1}}{\partial \phi_{1}}+\frac{\partial \mu_{1}}{\partial \phi_{2}} \cdot \frac{d \phi_{2}}{d \phi_{1}}\right) \cdot \frac{d \phi_{1}}{d m} \\
& J_{2}=-\frac{D_{2}}{R T} \cdot \phi_{2} \cdot\left(\frac{\partial \mu_{2}}{\partial \phi_{2}}+\frac{\partial \mu_{2}}{\partial \phi_{1}} \cdot \frac{d \phi_{1}}{d \phi_{2}}\right) \cdot \frac{d \phi_{2}}{d m}
\end{aligned}
$$

Equation (12) together with the steady-state assumption and one of the boundary conditions yields the concentration profiles $\phi_{1}(m, t)$ and $\phi_{2}(m, t)$. Since there is no analytical solution for the coupled differential equation (12), we represent the solution as follows:

$$
\begin{aligned}
& \phi_{1}(m, t)=F_{1}\left(m, t, D_{1}, D_{2}, J_{1}, J_{2}, \mu_{1}, \mu_{2}\right) \\
& \phi_{2}(m, t)=F_{2}\left(m, t, D_{1}, D_{2}, J_{1}, J_{2}, \mu_{1}, \mu_{2}\right)
\end{aligned}
$$

In this way $\phi_{1}$ and $\phi_{2}$ are expressed as functions of all these parameters. With respect to time $t=$ 0 the change in nonsolvent and solvent content per unit area of membrane at time $t=t_{1}$ is, respectively,

$$
\Delta V_{1}=\int_{0}^{m_{d}\left(t_{1}\right)}\left(\frac{\phi_{1}\left(m, t_{1}\right)}{\phi_{3}\left(m, t_{1}\right)}-\frac{\phi_{1}^{c}}{\phi_{3}^{c}}\right) \cdot d m
$$

and

$$
\Delta V_{2}=\int_{0}^{m_{d}\left(t_{1}\right)}\left(\frac{\phi_{2}\left(m, t_{1}\right)}{\phi_{3}\left(m, t_{1}\right)}-\frac{\phi_{2}^{c}}{\phi_{3}^{c}}\right) \cdot d m
$$

The result is

$$
\Delta V_{1} / \Delta V_{2}=F_{3}\left(m, t_{1}, D_{1}, D_{2}, J_{1}, J_{2}, \mu_{1}, \mu_{2}\right)
$$

On the other hand, the concentration distribution as described by eqs. (13) is generated by the nonsolvent and solvent fluxes through the interface, so the quantities $\Delta V_{1}$ and $\Delta V_{2}$ must be equal to $-\int_{0}^{t_{1}} J_{1}(t) d t$ and $-\int_{0}^{t_{1}} J_{2}(t) d t$, respectively, where $J_{1}(t) / J_{2}(t)=\sigma$ at every time, and therefore

$$
\frac{\Delta V_{1}}{\Delta V_{2}}=\int_{0}^{t} J_{1}(t) \cdot d t / \int_{0}^{t} J_{2}(t) \cdot d t=\sigma
$$

Comparing eqs. (15) and (16), we see that this condition is not fulfilled. Equations (15) and (16) are identical only if the concentration profiles are computed under the restriction of eqs. (4) and (5). From Eqs. (12) and (15) it can be seen that in the Cohen, Tanny, and Prager description the ratio $\Delta V_{1} / \Delta V_{2}$ depends on the interaction parameters that appear in the Flory-Huggins expressions for the chemical potentials.

\section{DISCUSSION}

It has been pointed out above that the $\phi_{1}$ vs. $\phi_{2}$ curve, calculated with the aid of eq. (11) and characteristic for a certain ratio $\sigma$ (see Fig. 2), cannot give the correct concentration distribution in a film which is subject to a nonsolvent-solvent exchange with the same ratio $\sigma$. The consequence

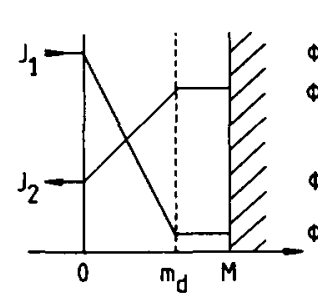

(a)

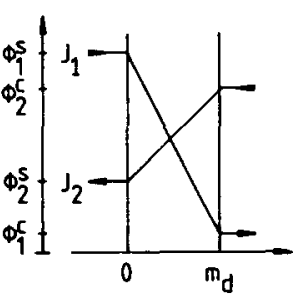

(b)

Fig. 2. Concentration profiles: (a) concentration distribution in a cast polymer film, when the diffusion boundary has reached $m_{d}$; (b) steady-state concentration distribution in a sheet with thickness $m_{d}$. 
is that the composition paths drawn in the ternary phase diagrams by Cohen, Tanny, and Prager are not valid. This can be illustrated by two peculiar features of the composition paths shown in Figures 3-5 of their paper and also in our Figure 3. Figure 3 has been taken from the thesis of Altena, ${ }^{11}$ who used in Eq. (11) the following expressions for the chemical potentials ${ }^{10}$ :

$$
\begin{gathered}
\left(\mu_{1}-\mu_{1}^{0}\right) / R T=\ln \left(\phi_{1}\right)-s \cdot \phi_{2}-r \cdot \phi_{3} \\
+\left(1+\chi_{12} \cdot \phi_{2}+\chi_{13} \cdot \phi_{3}\right) \cdot\left(1-\phi_{1}\right)-s \chi_{23} \phi_{2} \phi_{3} \\
\left(\mu_{2}-\mu_{2}^{0}\right) / R T=s \cdot \ln \left(\phi_{2}\right)-\phi_{1}-\mathrm{r} \cdot \phi_{3} \\
+\left(s+\chi_{12} \cdot \phi_{1}+s \cdot \chi_{23} \cdot \phi_{3}\right) \cdot\left(1-\phi_{2}\right)-\chi_{13} \cdot \phi_{1} \cdot \phi_{3}
\end{gathered}
$$

The chemical potentials are expressed per mole of segments, one mole of segments having the same volume as one mole of species 1 . The $\chi_{i j}$ parameters are the interaction parameters of components $i$ and $j ; s$ and $r$ are $\nu_{1} / \nu_{2}$ and $\nu_{1} / \nu_{3}$, the ratios of the molar volumes.

From Figure 3 it can be seen that (i) the location of the composition path at a constant ratio $\sigma$ depends on the thermodynamic properties of the system and that (ii) the average polymer content does increase for $\sigma=-1$.

Although the interaction parameters will have some influence on the concentration distribution, it is clear that the average change in composition in the film should be solely determined by the fluxes $J_{1}$ and $J_{2}$. Furthermore, it is obvious that if $\sigma=-1$, i.e., if the volume of solvent leaving is replaced by an equal volume of nonsolvent entering, the average polymer concentration should remain constant. In fact, the latter has been stated implicitly by Cohen, Tanny, and Prager themselves in deriving their eq. (10). ${ }^{6}$ This equation, the result of the mass balance in time, reads

$$
\int_{0}^{t}\left(J_{1}+J_{2}\right) \cdot d t=\int_{0}^{m_{d}}\left(\frac{1}{\phi_{3}^{c}}-\frac{1}{\phi_{3}}\right) \cdot d m
$$

If the ratio $\sigma$ has the value -1 , which means $J_{1}+J_{2}=0$, this equation implies that $\bar{\phi}_{3}=\phi_{3}^{\mathrm{c}}$.

Since eq. (11) cannot be applied to our diffusion problem, we have to return to eqs. (3) and (4), which describe the diffusion phenomenon-together with the conditions (5)-(7). The use of boundary condition (6) presumes equilibrium between the nonsolvent bath and the film surface. In case the bath is not well stirred, Cohen, Tanny, and Prager suggest the use of finite transfer coefficients within a boundary layer, which will influence the surface concentrations.

In our opinion, even if there is no concentration gradient in the bath, there will be another resistance to the mass transport, i.e., the resistance in the film/bath interface itself. Thus, eq. (6) is replaced by

$$
\begin{gathered}
m=0: \quad \frac{D_{1} \cdot \phi_{1}}{R T} \cdot \frac{\partial \mu_{1}}{\partial m}=-J_{1}(0, t)=L_{1} \cdot \Delta \mu_{1} \\
\frac{D_{2} \cdot \phi_{2}}{R T} \cdot \frac{\partial \mu_{2}}{\partial m}=-J_{2}(0, t)=L_{2} \cdot \Delta \mu_{2}
\end{gathered}
$$

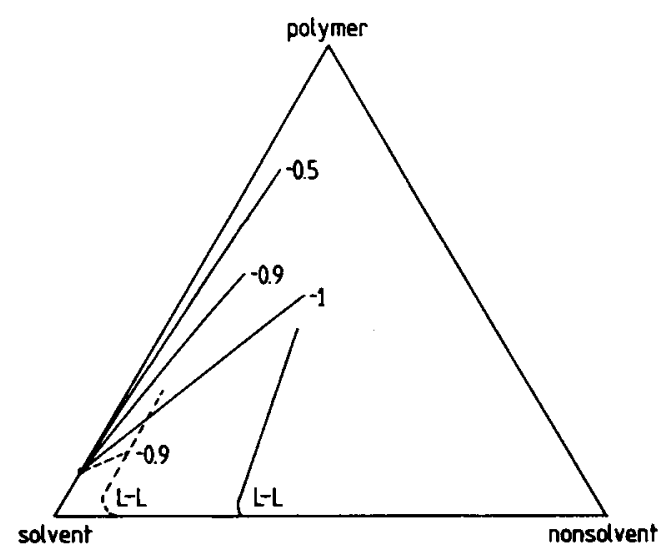

Fig. 3. Composition paths for various values of $\sigma$ in the ternary polymer/solvent/nonsolvent system. Parameter values: $s=0.2 ; r=0.002 ; \chi_{13}=1.5, \chi_{23}=0.0$, and $\chi_{12}=1.0(-)$ or $\chi_{12}=0.0(--)$. The binodals for liquid-liquid phase separation have been calculated. ${ }^{11,12}$ 
$L_{1}$ and $L_{2}$ are the permeability coefficients for nonsolvent and solvent in the interface. The potential difference $\Delta \mu_{i}$ is the difference at the interface:

$$
\begin{gathered}
\Delta \mu_{i}=\mu_{i, \text { casting solution, } m=0}\left(\phi_{1}, \phi_{2}\right) \\
-\mu_{i, \text { coagulation bath, } m=0}\left(v_{1}, v_{2}\right)
\end{gathered}
$$

The volume fractions of nonsolvent and solvent in the bath are $v_{1}$ and $v_{2}$. We have used similar relation to describe the volume fluxes through the interface at the first instance of coagulation. ${ }^{13}$ Equations (3)-(5), (7), and (19) together describe the diffusion during immersion precipitation and they can be solved numerically in order to yield the concentration profiles in the polymer film as a function of time (see Appendix B for a specific example). In this way it is possible to evaluate the influence of the transport parameters $L_{i}$ and $D_{i}$; this work is in progress. From eq. (19) it can already be deduced that the ratio $J_{1}(0, t) / J_{2}(0, t)$ in general is not independent of time. In our opinion this is very important with respect to the formation of asymmetric structures in the film. ${ }^{8,11}$

\section{APPENDIX A}

The mass conservation law is applied to the differential volume element shown in Figure 4 . For the accumulation of species $i$ we write

$$
A \cdot\left(\phi_{i} \cdot \Delta z\right)_{t+\Delta t}-A \cdot\left(\phi_{i} \cdot \Delta z\right)_{t}=A \cdot \Delta m \cdot\left(\frac{\phi_{i}}{\phi_{3}}\right)_{t+\Delta t}-A \cdot \Delta m \cdot\left(\frac{\phi_{i}}{\phi_{3}}\right)_{t}=\frac{\partial\left(\phi_{i} / \phi_{3}\right)}{\partial t} \cdot \Delta m \cdot \Delta t \cdot A
$$

Flow of species $i$ in minus flow of species $i$ out can be expressed as

$$
A \cdot \Delta t \cdot\left(J_{i}\right)_{m}-A \cdot \Delta t \cdot\left(J_{i}\right)_{m+\Delta m}=-\frac{\partial J_{i}}{\partial m} \cdot \Delta m \cdot \Delta t \cdot A
$$

Thus

$$
\frac{\partial\left(\phi_{\mathrm{i}} / \phi_{3}\right)}{\partial \mathrm{t}}=-\frac{\partial J_{i}}{\partial m}
$$

\section{APPENDIX B}

The diffusion problem as described by eqs. (3) - (5), (7), and (19) has been solved for the case

$$
J_{1}(0, t)=-J_{2}(0, t)=\text { const }
$$

This boundary condition has been chosen to permit us to compare the computed concentration profile with the concentration path calculated with the aid of eq. (11) for $\sigma=-1$ (see Fig. 3). The numerical procedure used is the NAG library routine $\mathrm{D} 03 \mathrm{PGF}^{14}$ and the parameters in the equations had the following values:

$$
\begin{aligned}
J_{1}(0, t) & =2 \times 10^{-5} \mathrm{~m} / \mathrm{s} \\
J_{2}(0, t) & =-2 \times 10^{-5} \mathrm{~m} / \mathrm{s} \\
\phi_{1}^{c} & =0.0001 \\
\phi_{2}^{c} & =0.7999 \\
\phi_{3}^{c} & =0.2000 \\
Z & =1.10^{-4} \mathrm{~m}
\end{aligned}
$$

$$
\begin{aligned}
D_{1} & =2 \times 10^{-9} \mathrm{~m}^{2} / \mathrm{s} \\
D_{2} & =5 \times 10^{-9} \mathrm{~m}^{2} / \mathrm{s} \\
\chi_{12} & =0.0 \\
\chi_{13} & =1.5 \\
\chi_{23} & =0.0 \\
\mathbf{s} & =0.2 \\
\mathbf{r} & =0.002
\end{aligned}
$$

The concentration profile and the concentration path at time $t=0.1 \mathrm{~s}$ are displayed in Figure 5.

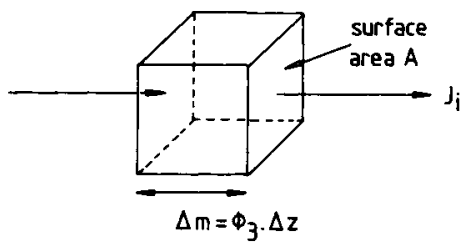

Fig. 4. Differential volume element. 


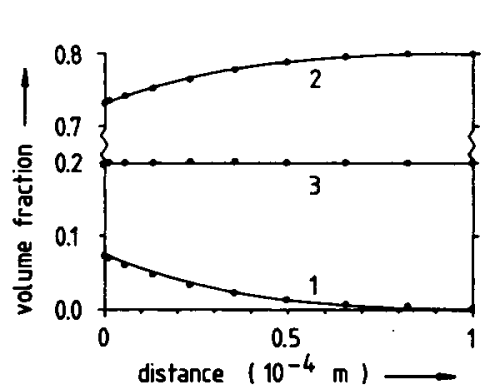

a)

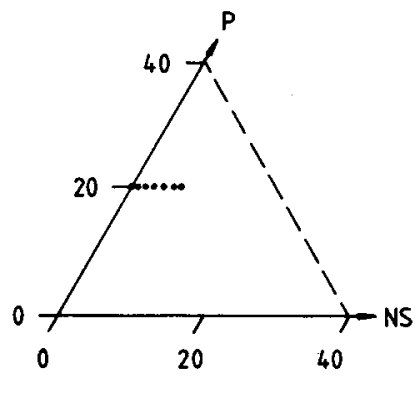

b)

Fig. 5. Concentration profile (a) and concentration path (b) calculated with the aid of eqs. (3)-(5), (7), (19), and (B1).

From this figure it appears that the overall polymer concentration in the film has not changed in the case $\sigma=-1$, as would be expected.

\section{References}

1. H. Strathmann, P. Scheible, and R. W. Baker, J. Appl. Polym. Sci., 15, 811 (1971).

2. M. A. Frommer and D. Lancet, in Reverse Osmosis Research, H. K. Lonsdale and H. E. Podall, Eds., Plenum, New York, 1972.

3. M. Guillotin, C. Lemoyne, C. Noël, and L. Monnerie, Desalination, 21, 165 (1977).

4. H. Strathmann and K. Koch, Desalination, 21, 241 (1977).

5. D. M. Koenhen, M. H. V. Mulder, and C. A. Smolders, J. Appl. Polym. Sci., 21, 199 (1977).

6. C. Cohen, G. B. Tanny, and S. Prager, J. Polym. Sci. Polym. Phys. Ed., 17, 477 (1979).

7. C. A. Smolders, in Ultrafiltration Membranes and Applications, A. R. Cooper, Ed., Plenum, New York, 1980.

8. H. Bokhorst, F. W. Altena, and C. A. Smolders, Desalination, 38, 349 (1981).

9. G. S. Hartley and J. Crank, Trans. Faraday Soc., 45, 801 (1949).

10. P. J. Flory, Principles of Polymer Chemistry, Cornell University, Ithaca, NY, 1953.

11. F. W. Altena, Thesis, Twente University of Technology, 1982, Chap. 7.

12. F. W. Altena and C. A. Smolders, in F. W. Altena's thesis, Twente University of Technology, Enschede, 1982, Chap. 5. [Macromolecules, 15, 1491 (1982)].

13. J. G. Wijmans, J. P. B. Baaij, and C. A. Smolders, J. Membr. Sci., 14, 263 (1983).

14. P. M. Dew and J. E. Walsh, Numerical Analysis Report No. 49, University of Manchester, 1980.

J. G. WIJMANS

F. W. ALTENA

C. A. SMOLDERS

Twente University of Technology

Department of Chemical Technology

P.O. Box 217, Enschede, The Netherlands

Received February 7, 1983

Accepted October 17, 1983 